\title{
Performance Analysis of Image Denoising Technique using Neural Network
}

\author{
Shrish Pathak \\ M.Tech.Scholar ECE \\ Department SHIATS-DU \\ Allahabad, U.P., India
}

\author{
Mukesh Kumar \\ Assistant Professor \\ ECE \\ Department SHIATS-DU \\ Allahabad, U.P., India
}

\author{
A.K.Jaiswal \\ Professor,H.O.D. ECE \\ Department SHIATS-DU \\ Allahabad, U.P., India
}

\author{
Rohini Saxena \\ Assistant Professor \\ ECE \\ Department SHIATS-DU \\ Allahabad, U.P., India
}

\begin{abstract}
ABSTRCT
Image processing is widely applied in various area of applications such as Medical, military, agriculture, etc.. The problem which generally occurs in image processing is the removal of noise generated due to various sources. In this paper a new approach based on neural network technique is proposed for the removal of noise. This technique follows three levels. This technique combines the advantages of filtering, neural network and bayes shrinkage technique. The noisy image is first passed through a bilateral filter and neural network is applied to the filtered image and the output of $\mathrm{NN}$ is then applied to bayes shrink. The proposed method outperforms other methods both visually and in case of objective quality peak-signal-to-noise ratio (PSNR) and MSE. Proposed method is verified for additive white Gaussian noise.
\end{abstract}

\section{Keywords}

Image denoising, wavelet threshoding, Bayes threshold, Multiscale product threshold, PSNR, MSE, DWT, IDWT

\section{INTRODUCTION}

Digital imaging is widely used in all areas related with our daily life. Because of increasing population advance imaging algorithms are required. Normally a digital image is corrupted by noise generated from various sources. There are various sources of noise in a digital image. For example, dark current noise is due to the thermally generated electrons, Shot noise is due to the quantum uncertainty in photoelectron generation .Quantization and amplifier noise occurs during the conversion of the number of electrons generated to pixel intensities. The noise characteristics in an image depends on various factors, including sensor type, pixel dimensions, temperature, exposure time, and ISO speed [7]-[14]-[15]. Various image denoising techniques has been proposed by various researchers over the past few decades; among all these techniques, wavelet thresholding is one of the most widely used techniques. In wavelet thresholding, a signal is decomposed into its approximation (low-frequency) and detail (high-frequency) subbands; since most of the image information is concentrated in a few large coefficients, the detail subbands are processed with appropriate thresholding operations. The prominent task in wavelet thresholding is the selection of threshold. Various threshold selection techniques have been proposed, for example, VisuShrink [2], SureShrink [3], and BayesShrink [4]. In the VisuShrink approach, a universal threshold that is a function of the noise variance and the number of samples is developed based on the minimax error measure. In the SureShrink approach, threshold value is optimal in terms of the Stein's unbiased risk estimator. The threshold value is selected in a Bayesian framework, through modeling the distribution of the wavelet coefficients as Gaussian, in BayesShrink approach. Several advancement have been proposed in the shrinkage algorithms by considering interscale and intrascale correlations of the wavelet coefficients [8]-[10]-[16]. The paper is organized in the following behavior, in Section II wavelet transform is explained, in Section III Neural network. In section IV a new method for image denoising is proposed and compared in Section V based on performance metric and finally work in concluded in Section VI.

\section{WAVELET THRESHOLDING}

MULTISCALE decompositions have shown significant advantages in the representation of signals, and they are used very frequently in image processing applications such as image denoising, image compression and image segmentation [4]. The elimination of noise from a natural image corrupted by Gaussian noise is a very serious problem in image processing. The wavelet transform has become a useful tool in image denoising due to its energy compaction capacity. According to it the wavelet transform yields a large number of small coefficients and a small number of large coefficients. Image denoising algorithms that use the wavelet transform consist of three steps as.

1) Calculate the wavelet transform of the noisy signal.

2) Modify the noisy wavelet coefficients according to some rule.

3) Compute the inverse transform using the modified coefficients.

Several rules related to second step are available One of the most well-known rules is soft thresholding analyzed by Donoho [1]. Because of to its effectiveness and simplicity, it is frequently used in the literature by various researchers. The main idea is to subtract the threshold value from all coefficients larger than and to set all other coefficients to zero.

Let $\boldsymbol{f}=\{f i j, \mathrm{i}, \mathrm{j}=1,2 \ldots \mathrm{N}\}$ denote the $\mathrm{M} \times \mathrm{M}$ matrix of the original image to be recovered and $\mathrm{M}$ is some integer power of 2. During transmission the $f$ is corrupted by white Gaussian Noise $n i j$ with standard deviation $\sigma$. At the receiver side, the

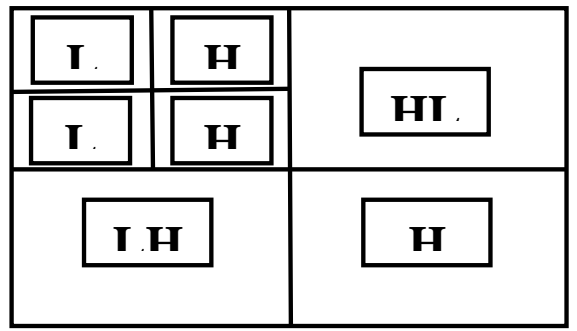

Figure 1: Wavelet Transform 
noisy received signal $g i j=f i j+\sigma n i j$ is obtained. The goal is to estimate the signal $\boldsymbol{x}$ from noisy observations gij such that Mean Squared error is minimum. Let $\mathrm{W}$ and $\mathrm{W}^{-1}$ denotes the two dimensional orthogonal discrete wavelet transform (DWT) matrix and its inverse respectively. $\mathrm{G}=\mathrm{Wg}$ represents the matrix of wavelet coefficients of $g$ having four subbands (LL, LH, HL and $\mathrm{HH}$ ). The sub-bands $\mathrm{HH}_{1}, \mathrm{HL}_{1}, \mathrm{LH}_{1}$ are called details, where 1 is the scale varying from $1,2 \ldots \ldots . \mathrm{J}$ and $\mathrm{J}$ is the total number of decompositions. The size of the subband at scale 1 is N/2 $\times N / 2^{1}$ . The subband LLJ is the low-resolution residue [1].

The wavelet thresholding denoising method processes each coefficient of $\mathrm{G}$ from the detail subbands with a soft threshold function to obtain $P$. The denoised estimate is inverse transformed to $\boldsymbol{x}=\boldsymbol{W}^{-1} \boldsymbol{P}[11]-[12]$.

\section{NEURAL NETWORK}

Artificial Neural Network (ANN) is a method used in information processing by the researchers. The basic concept of ANN is inspired by the biological nervous systems, such as the brain. It is composed of a large number of highly interconnected processing elements these elements are known as neurons. ANNs is trained by training sets provided to the system [18]. An ANN is configured for a specific application, such as pattern recognition or data classification, through a learning process. Learning in biological systems involves adjustments to the synaptic connections that exist between the neurons. Learning a dictionary is sometimes accomplished through learning on a noise-free dataset. There are two types of network:

Feed-forward networks: Feed-forward ANNs is technique in which signal flows in only one way from input to output. There is no feedback path (loops) available i.e. the output of any layer does not affect that same layer.

Feed-backward networks: Feedback network is a technique in which signal flows in both directions by introducing loops in the network. Feedback networks are very powerful and can get extremely complicated. Feedback networks are dynamic system. Its state changes continuously until it reach an equilibrium point. They remain at the equilibrium point until the input changes and a new equilibrium needs to be found. Feedback architectures are also referred to as interactive or recurrent, although the latter term is often used to denote feedback connections in single-layer organizations.

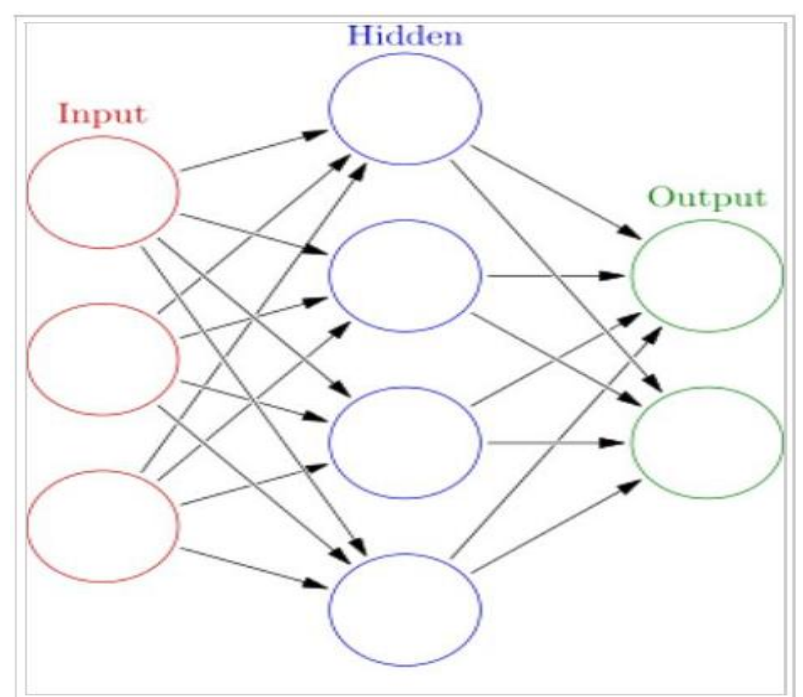

Figure 2: Basic Neural Network
In this paper we have used MLP. MLP stands for multi-layer perceptron (MLP). MLP is also called feed-forward neural network. This is a nonlinear function that maps vector-valued input via several hidden layers to Vector-valued output. For instance, an MLP with two hidden layers can be written as,

$$
P(k)=a_{3}+W_{3} \tanh \left(a_{2}+W_{2} \tanh \left(a_{1}+W_{1} * k\right)\right)
$$

Where $W_{1}, W_{2}, W_{3}$ are weight matrices and $a_{1}, a_{2}, a_{3}$ are vectorvalued biases. The function Tanh operates component-wise. The architecture of an MLP is defined by the number of hidden layers and by the layer sizes.

\section{PROPOSED DENOISING METHOD}

In this paper we have proposed a new image denoising technique based on Neural Network technique. First the received noisy image is applied to a filter and output of filter is applied to neural network and then a bayes shrink is used for removal of noise in the final step as shown in figure 3 .

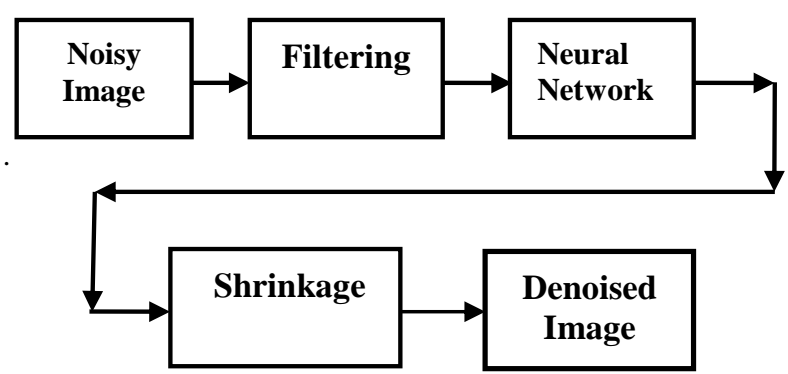

Figure 3: Block Diagram of Proposed Method

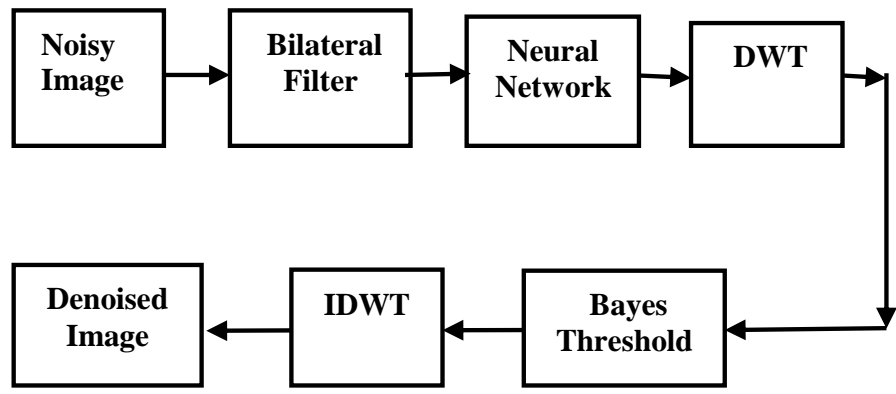

Figure 4: Detailed Block Diagram of Proposed Method

Figure 4 explains the proposed method in detail. As shown in figure in first stage filtering is done by bilateral filter and neural network is applied, then discrete wavelet transform is taken and result is thresholded using Bayesian approach because Bayesian threshold has the better performance in comparison to normal and visu threshold method. After applying threshold inverse discrete wavelet transform is taken. After inverse discrete wavelet transform we will get the final image with less noise. 
Table 1. Comparison with various techniques

\begin{tabular}{|c|c|c|c|c|c|c|}
\hline \multirow[t]{2}{*}{ Technique } & \multicolumn{3}{|c|}{ Noise Density $\sigma=14$} & \multicolumn{3}{|c|}{ Noise Density $\sigma=18$} \\
\hline & PSNR & MSE & Max Error & PSNR & MSE & Max Error \\
\hline Noisy Image & 25.21 & 195.77 & 55.45 & 22.94 & 330.33 & 75.90 \\
\hline BayesShrink & 29.18 & 78.45 & 48.82 & 27.56 & 115.87 & 63.46 \\
\hline Bilateral & 25.21 & 195.75 & 55.45 & 22.94 & 330.32 & 75.90 \\
\hline Multiscale Product Thresholding & 29.37 & 75.07 & 57.15 & 27.98 & 103.49 & 63.74 \\
\hline Adaptive Spatial Wavelet Threshold & 28.21 & 98.02 & 63.49 & 26.47 & 146.37 & 74.97 \\
\hline \multirow[t]{3}{*}{ Proposed } & 29.22 & 77.65 & 54.69 & 29.23 & $\mathbf{7 7 . 5 7}$ & 54.57 \\
\hline & \multicolumn{3}{|c|}{ Noise Density $\sigma=22$} & \multicolumn{3}{|c|}{ Noise Density $\sigma=30$} \\
\hline & PSNR & MSE & Max Error & PSNR & MSE & Max Error \\
\hline Noisy Image & 21.38 & 473.19 & 89.99 & 18.62 & 893.59 & 120.99 \\
\hline BayesShrink & 26.63 & 141.33 & 63.55 & 24.89 & 211.07 & 94.44 \\
\hline Bilateral & 21.38 & 473.19 & 89.98 & 18.62 & 893.60 & 120.99 \\
\hline Multiscale Product Thresholding & 26.86 & 134.02 & 90.33 & 25.43 & 186.42 & 103.61 \\
\hline Adaptive Spatial Wavelet Threshold & 25.54 & 181.65 & 84.34 & 23.62 & 282.29 & 96.09 \\
\hline Proposed & 29.04 & 81.16 & 54.85 & 27.41 & 118.03 & 61.01 \\
\hline
\end{tabular}

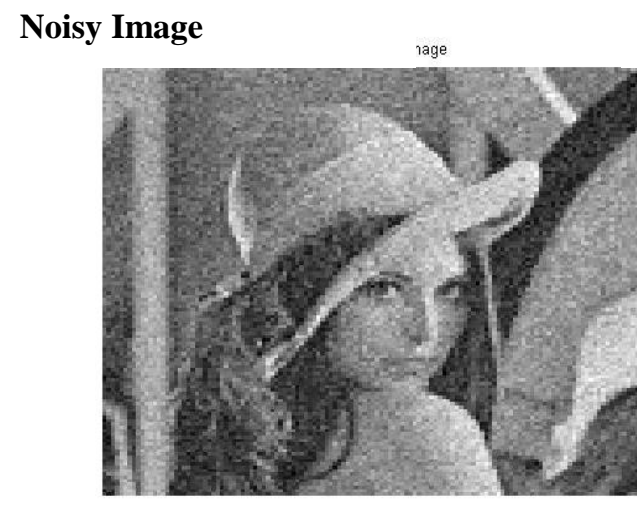

Bilateral Filter

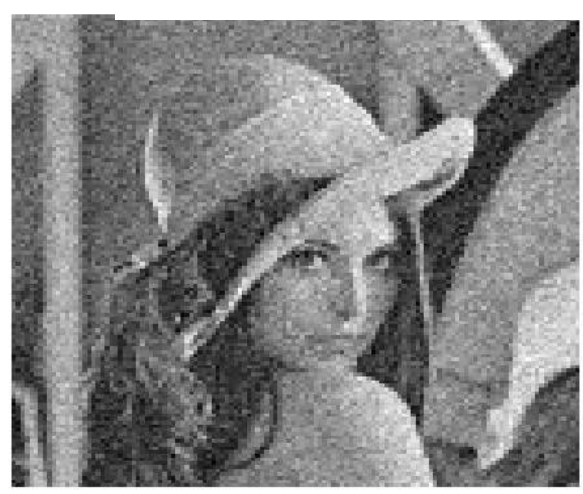

Bayes Shrinkage

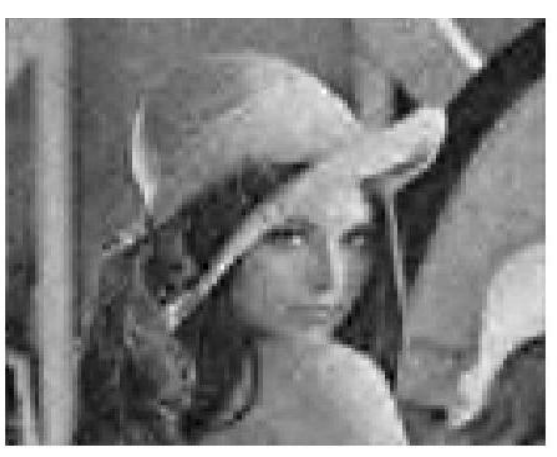

Multiscale Product Threshold

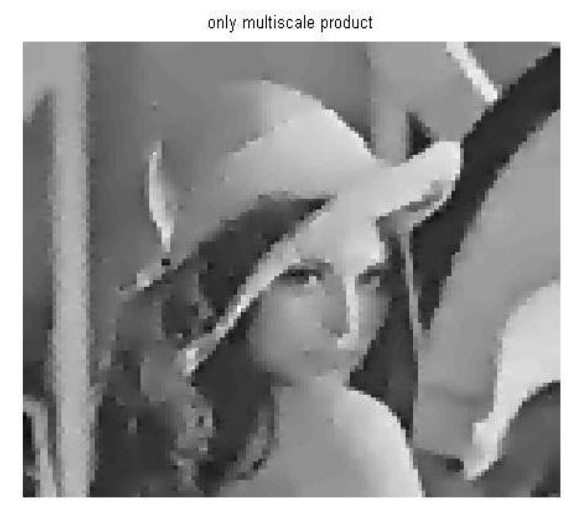


Adaptive Spatial Threshold

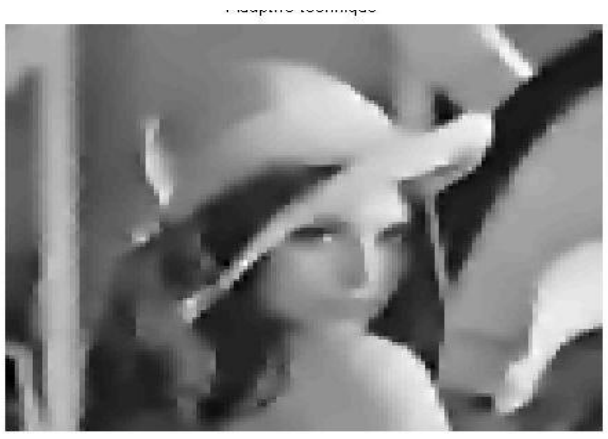

Proposed Method

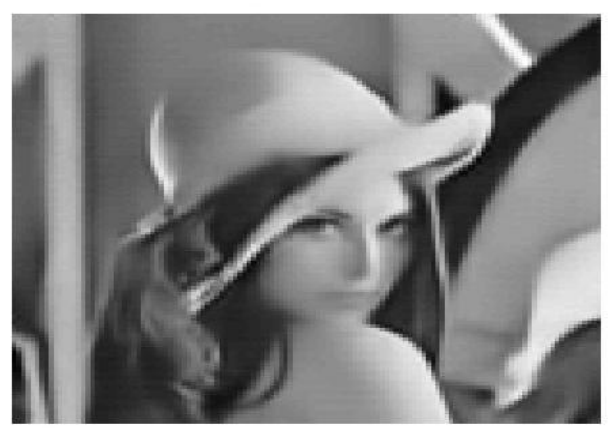

Figure 5: Result comparison of with existing method

\section{COMPARISON WITH EXISTING WORK}

The proposed denoising method is compared with existing techniques based on MSE (Mean square error) and PSNR (Peak signal to noise ratio) and Maximum error. The Mean Square Error (MSE) and the Peak Signal to Noise Ratio (PSNR) are the two globally used error metrics to compare image quality. The MSE represents the cumulative squared error between the noisy and the original image, whereas PSNR represents a measure of the peak error. The lower the value of MSE, lower the error. To compute the PSNR, the block first calculates the mean-squared error using the equation:

$$
M S E=\frac{1}{M N} \sum_{m=1}^{M} \sum_{n=1}^{N}(x(m, n)-\hat{x}(m, n))^{2}
$$

In the previous equation, $M$ and $N$ are the number of rows and columns in the input images, respectively. Then the block computes the PSNR using the following equation:

$$
P S N R=10 \log \frac{R^{2}}{M S E}
$$

In the equation (3), $R$ is the maximum fluctuation in the input image. For example, if the input image has a double-precision floating-point data type, then $R$ is 1 . If it has an 8-bit unsigned integer data type, $R$ is 255 , etc. Table- 1 compares various techniques on the basis of their PSNR, MSE and Max Error Gaussian noise. Table contains the values for different noise densities. Figure 4 shows the result for all the techniques. After the analysis of proposed scheme it is observed that it has better performance for Gaussian noise in for larger values of noise densities The result is simulated using Matlab.

\section{CONCLUSION}

This work incorporates an adaptive technique which combines bilateral filter, neural network and Bayes Shrinkage thresholding. The advantage of proposed technique is that it has not only improvement in image characteristics such as PSNR \& MSE but also in image perception (Visual image). This technique is also less complex in terms of level of denoising in comparison to adaptive spatial multiscale technique. The proposed technique is verified for Gaussian noise and observed that it has better performance for when the noise density is higher for low noise density this method has moderate performance.

\section{REFERENCES}

[1] I. Daubechies, "Ten Lectures on Wavelets". Philadelphia, PA: SIAM,

[2] D.L.Donoho and I. M. Johnston, (1994) "Ideal spatial adaptation by wavelet shrinkage".
[3] D. L. Donoho, I. M. Johnston, G. Kerkyacharian, and D. Picard, (1995) "Wavelet shrinkage: Asymptopia?".

[4] S. G. Chang, B. Yu and M. Vetterli, (2000) "Adaptive wavelet thresholding for image denoising and compression".

[5] R.C. Gonzalez and R.E. Woods, (2002) "Digital Image Processing".

[6] M.Vetterli and C. Herley, (1992) "Wavelet and filter banks: Theory and design".

[7] M.K.Mihcak, I. Kozintsev and K.Ramchandran, (1999) "Spatially Adaptive Statistical Modeling of Wavelet Image Coefficients and its Application to Denoising".

[8] Ming Zhang and Bahadir K. Gunturk( 2008)"Multiresolution Bilateral Filtering for Image Denoising,".

[9] D.L.Donoho, (1995)"Denoising and soft thresholding,".

[10] P. Bao and L. Zhang, (2003). "Noise reduction for magnetic resonance images via adaptive multiscale products thresholding,".

[11] A. Pizurica, W. Philips, I. Lemahieu, and M. Acheroy, (2003) "A versatile wavelet domain noise filtration technique for medical imaging,".

[12] L. Sendur and I. W. Selesnick, ( 2002)"Bivariate shrinkage functions for wavelet-based denoising exploiting interscale dependency,".

[13] Q. Pan et al., (1999) "Two denoising methods by wavelet transform,".

[14] S. M. Smith and J. M. Brady, (1997) "Susan-A new approach to low level image processing,".

[15] A. Macovski, (1996) "Noise in MRI".

[16] F. Luisier, T. Blu, and M. Unser, (2007) "A new sure approach to image denoising: Inter-scale orthonormal wavelet thresholding,".

[17] Junyuan Xie, Linli Xu and Enhong Chen(2012) "Image Denoising and Inpainting with Deep Neural Networks".

[18] G. Hinton, S. Osindero, and Y. Teh. (2006) "A fast learning algorithm for deep belief nets".

[19] K. Hornik, M. Stinchcombe, and H. White. (1989) "Multilayer feed forward networks are universal approximators" 